\title{
Les réserves de biosphère du Canada : apprentissage social et bonnes pratiques
}

Hélène Godmaire, Maureen Reed et Marc-André Guertin

\author{
(2) OpenEdition \\ Journals \\ Édition électronique \\ URL : http://journals.openedition.org/ere/807 \\ DOI : $10.4000 /$ ere.807 \\ ISSN : 2561-2271 \\ Éditeur \\ Centr'ERE \\ Référence électronique \\ Hélène Godmaire, Maureen Reed et Marc-André Guertin, « Les réserves de biosphère du Canada : \\ apprentissage social et bonnes pratiques », Éducation relative à l'environnement [En ligne], Volume 11 | \\ 2014, mis en ligne le 29 mai 2018, consulté le 21 février 2020. URL : http://journals.openedition.org/ \\ ere/807 ; DOI : 10.4000/ere.807
}




\title{
Les réserves de biosphère du Canada : apprentissage social et bonnes pratiques
}

\author{
Hélène Godmaire, Maureen Reed et Marc-André Guertin
}

\section{NOTE DE L'AUTEUR}

Ce texte est issu d'une recherche en cours à l'École de l'environnement et de la viabilité de l'Université de la Saskatchewan. Les références et le langage utilisés peuvent ne pas être familiers aux lecteurs des domaines de l'ERE et l'EDD. Toutefois, ils peuvent être d'intérêts pour ouvrir de nouveaux horizons sur des approches et des enjeux de recherche qui convergent vers des prises de décision et d'actions réfléchies à l'égard de l'environnement et des communautés.

1 De l'échelle locale à l'échelle mondiale, les demandes sur les écosystèmes ne cessent de s'accroître alors que la capacité de ces derniers à fournir des biens et services de qualité diminue. Ces transformations, exacerbées par la situation économique mondiale et l'accroissement des inégalités sociales, soulèvent inexorablement le défi d'améliorer les savoir-faire et les savoir-être à l'égard de l'environnement et des systèmes de gouvernance, à tous les niveaux de compétences. L'atteinte d'un équilibre entre la capacité de support des écosystèmes et les besoins des êtres humains sera essentielle pour assurer la viabilité. En réponse aux changements rapides et souvent irréversibles, des efforts orientés sur la compréhension des mécanismes de conscientisation, de mobilisation et de gouvernance intégrée sont en cours dans de nombreux endroits à travers le monde. Les réserves de biosphère en sont un exemple. Ces lieux sont des régions écologiques exceptionnelles reconnues par l'UNESCO. Elles sont issues du Programme L'Homme et la biosphère (MAB - Man and the Biosphere) de l'UNESCO. Lancé au début des années 1970, le programme MAB fait appel à de nombreuses disciplines scientifiques pour améliorer la relation entre les personnes et l'environnement. Fondées sur ce programme international, les réserves de biosphère 
intègrent les dimensions écologique, culturelle, sociale et économique de la vie d'une région donnée et son paysage. Grâce à son réseau de réserves de biosphère, le programme $\mathrm{MAB}$ favorise également le partage des connaissances, de la recherche, de la surveillance écologique, de l'éducation, de la formation et de la prise de décision participative. Le travail des réserves de biosphères est guidé par la Stratégie de Séville et le cadre statutaire de 1995 qui exige un examen périodique une fois tous les dix ans. En outre, le programme MAB établit un plan stratégique tous les cinq ans. Actuellement, c'est le Plan d'action de Madrid qui est mis en œuvre (2008-2013).

2 Les réserves de biosphère abordent l'une des questions les plus difficiles à laquelle nous sommes confrontés aujourd'hui: comment faire pour maintenir l'intégrité des systèmes naturels tout en répondant aux besoins matériels des collectivités? Les réserves de biosphère ont pour mission de promouvoir la durabilité et la conservation en faisant appel à tous les intervenants. Elles stimulent la prise en charge des problématiques socio-environnementales par les communautés. Elles font appel à la recherche et au partage des connaissances afin de mieux informer les prises de décisions et l'élaboration des politiques en matière de développement durable. Le Canada compte 16 des 610 réserves de biosphère localisées dans 117 pays (UNESCO, 2013). Chaque réserve de biosphère définit ses propres priorités et ses actions de conservation de la diversité naturelle et culturelle et de développement responsable. Elles agissent à titre de régions modèles de l'engagement communautaire. Elles sont considérées comme des "labo-ratoires" d'apprentissage de la viabilité, de dialogue entre les praticiens et les chercheurs, supportées par l'expérimentation et le réseautage.

3 Le mandat des réserves de biosphère est vaste et exigeant. Du point de vue pratique, les réserves de biosphère sont souvent captives d'une course effrénée qui les mène de projet en projet, tout simplement pour survivre financièrement. Par conséquent, peu de réserves de biosphère canadiennes ont le temps d'évaluer leurs pratiques. Lorsque des innovations sont créées dans des réserves de biosphère, elles ne sont pas diffusées ni transférées; lorsque des projets ne parviennent pas à produire les résultats escomptés, ces apprentissages ne sont pas non plus partagés. Ainsi, chaque réserve de biosphère est vouée à réinventer la roue. L'occasion pour renforcer les capacités à travers le réseau est perdue, tout comme la possibilité pour les réserves de biosphère d'apprendre à être plus efficace en matière de conservation, conscientisation et gouvernance.

\section{Contexte : apprentissage et évaluation}

4 À l'échelle internationale, l'évaluation systématique des activités des réserves de biosphère est relativement récente (Stoll-Kleemann et Welp 2008; Schultz et Lundholm 2010; Stoll-Kleemann et al. 2010). Pourtant comme Bellamy et coll. (2001: 408) l'ont constaté : «L'évaluation est fondamentale pour identifier des voies d'action supportant une approche adaptative qui est suffisamment flexible pour répondre au défi du changement, tout en permettant un apprentissage progressif au niveau individuel, commu-nautaire, institutionnel et politique». Plummer et Armitage (2007) ont également noté que les évaluations sont indispensables pour mieux comprendre comment parvenir à la durabilité. Depuis la création des premières réserves de biosphère il y a 40 ans, de telles évaluations sont rares et n'intègrent pas toujours les 
facteurs contextuels comme les besoins des communautés locales (Conley et Moote, 2003). Le Canada suit la même tendance, il existe peu d'évaluations systématiques des apprentissages, des pratiques, des activités de recherche et des résultats de conservation (Schultz et Lundholm 2010; Reed, 2010). Les examens périodiques imposés (aux 10 ans) aux réserves de biosphère par le Programme L'Homme et la biosphère (MAB) de l'UNESCO rendent compte davantage de la conformité au programme que de la réussite ou échec des processus d'apprentissage (Reed et Egunyu, 2012). Ainsi, plusieurs interrogations demeurent. Par exemple: quelles sont les pratiques qui favorisent la viabilité ? Comment ces bonnes pratiques de gouvernance peuvent-elles être encouragées au-delà des réserves?

5 Les recherches sur le rôle des réserves de biosphère dans la gouvernance de l'environnement sont également récentes (Roots, 1989; Sian, 2000 ; Abrams, 2000 ; Dobell, 2002 ; Pollock, 2004 ; Francis, 2004; Whitelaw et coll., 2004; Reed, 2007; Jamieson et coll., 2008). La majeure partie des études portent sur des cas individuels, à petite échelle, et ne révèle pas les tendances communes, ni les leçons transférables (Abrams, 2000 ; Mendis, 2004 ; Whitelaw et coll., 2004 ; McCarthy et coll., 2006 ; Reed, 2007 ; Mendis-Millard et Reed, 2007 ; Pollock et coll., 2008 ; Pollock. 2009). Quoi qu'il en soit, ces études mettent en évidence le rôle que jouent certaines réserves de biosphère comme "intermédiaire et médiateur" entre les acteurs gouvernementaux et non gouvernementaux à l'échelle régionale (par exemple, Pollock et coll., 2008).

6 Au Canada, les premières réserves de biosphère ont été créées il y a plus de 30 ans, alors que les plus récentes ont été désignées au cours des dix dernières années. Dans ce contexte, l'expérience notable des plus anciennes et le dynamisme des plus récentes offrent une excellente occasion pour les membres du réseau d'apprendre les uns des autres. Toutefois, pour catalyser ces apprentissages un réseau de communication, une bonne connaissance des pratiques et un modus operandi de partage d'expériences sont nécessaires. Encore, l'évaluation des pratiques apparaît comme un dénominateur commun incontournable.

\section{Projet de réseaux et de stratégies d'apprentissage}

7 Le projet de Création de réseaux et de stratégies d'apprentissage dans les réserves de la biosphère canadiennes ${ }^{1}$, mené par l'Université de la Saskatchewan en collaboration avec l'Association canadienne des réserves de biosphère relève ces enjeux d'apprentissage et d'évaluation. Le projet vise à mobiliser les connaissances afin d'influencer l'évolution des pratiques et des politiques en matière de développement responsable. Il implique la participation des praticiens des réserves de biosphère, de chercheurs et d'étudiants universitaires, d'organisations nationales et de conseillers politiques qui sont invités à analyser et à réfléchir sur les expériences de ces organisations. Ce projet tente également de renforcer la capacité des réserves de biosphère canadiennes pour qu'elles contribuent plus efficacement à la gouvernance au sein de leurs régions et à l'évolution des stratégies de développement durable à l'échelle nationale et internationale. Sur le plan scientifique, cette proposition contribue à faire avancer les connaissances théoriques de la question de la gouvernance ${ }^{2}$ environnementale. Elle s'appuie sur l'apprentissage social, le réseautage, la mise en place d'une communauté de pratique, incluant la collaboration académique ainsi que l'identification et le développement de bonnes pratiques. 
8 Les objectifs du projet qui concernent plus particulièrement les savoirs sont les suivants :

1. Identifier collectivement, mettre en œuvre, et évaluer les bonnes pratiques en matière de gouvernance et de réseautage qui permettront aux réserves de biosphère de faire progresser la durabilité, d'apprendre les uns des autres, et d'échanger des connaissances à travers des échelles multiples.

2. Améliorer la recherche participative, l'échange d'information et le réseautage, entre les chercheurs universitaires, les réserves de biosphère, l'Association canadienne des réserves de biosphère, les comités nationaux et internationaux, les organisations internationales afin d'améliorer les possibilités d'apprentissage social et de développement durable.

3. Évaluer collectivement la façon dont le partenariat améliore la compréhension scientifique et les capacités pratiques des participants à s'engager dans la gouvernance et l'apprentissage de la durabilité.

\section{Éléments théoriques et méthodologiques}

9 Le projet s'appuie sur deux concepts qui se chevauchent : l'apprentissage social et la gouvernance environnementale. Le projet s'inspire de plusieurs recherches qui montrent l'importance d'intégrer des activités d'apprentissage au sein de structures de gouvernance afin de les rendre plus efficaces (Armitage et coll., 2008 ; Reed et coll., 2008 ; Pahl-Wostl, 2009 ; Schultz et Lundholm, 2010 ; Berkes, 2010 ; Löf, 2010 ; StollKleemann et coll., 2010). Certaines études soulignent « le rôle de l'apprentissage mutuel, compatible avec les objectifs de réserve de biosphère » (Stoll-Kleemann et Welp, 2008, p.166) et la nécessité pour ces organisations de s'engager dans des processus participatifs qui informent et impliquer les citoyens locaux dans tous les aspects d'opération et de gestion des réserves de biosphère (Lotze-Campen et al 2008 ; Stoll-Kleemann et Welp 2008; Schultz et Lundholm 2010). D'autres chercheurs qui s'intéressent à la capacité des réserves de biosphère à contribuer à la gouvernance régionale (Hockings et coll., 2006a, 2006b ; Bouamrane, 2007 ; Price et coll., 2010 ; Reed et Egunyu, 2013) suggèrent que des stratégies d'adaptation "for changing conditions » sont importantes. L'apprentissage et la coopération systématique peuvent fournir aux organisations une compréhension partagée des enjeux communs, les aider à faire face à l'incertitude et augmenter leur capacité à s'adapter aux défis en cours grâce à la rétroaction continue (Gunderson et coll., 2002 ; Folke et coll., 2003, 2005 ; Keen et coll., 2005 ; Plummer et Armitage, 2010). Fikret Berkes (2010) parle d'un cycle d'actionréflexion-action dans lequel les actions stratégiques sont révisées par l'évaluation et la rétroaction. Selon Berkes (2010: 7, traduction libre),

Chaque cycle commence par l'observation et l'identification des problèmes et des opportunités, ce qui conduit à l'action-réflexion et de nouveau à l'action. Les résultats des étapes successives doivent être évalués, suivis d'une réflexion, pour diriger le prochain cycle.

10 L'évaluation joue un rôle crucial, d'ailleurs son utilisation comme outil d'aide à l'apprentissage, à l'innovation et la gestion adaptative des aires protégées en général et des réserves de biosphère en particulier, soulève de plus en plus d'intérêt (Hockings et coll., 2006a, b ; Bouamrane, 2007 ; Reed et Egunyu, 2013). Cette tendance arrive juste, car il existe, en ce moment, un besoin urgent de partager des informations sur les pratiques dans les réseaux de réserves de biosphère à l'échelle nationale et 
internationale (Price et coll., 2010 ; Reed et Egunyu, 2013). Le Plan d'action de Madrid (2008-2013) l'inclut d'ailleurs dans ses cibles.

Dans le contexte de notre projet, l'exploration des bonnes pratiques (réflexion, autoévaluation) a été retenue comme outil d'apprentissage. Le terme bonne pratique correspond à «toutes les stratégies, plans, tactiques, processus, méthodologies, activités et approches qui sont documentés, accessibles, efficaces, pertinents [...], développés et mis en pratique par les organisations" (REM, 2012). Measham et coll. (2007) suggèrent que les pratiques de gestion optimales peuvent être regroupées en deux catégories principales, l'une associée à la régulation et l'autre associé à l'apprentissage et à l'adaptation. Notre intérêt se trouve ici dans le second, où les bonnes pratiques peuvent contribuer à favoriser l'amélioration et promouvoir l'apprentissage continu. Notre intérêt réside dans la façon d'utiliser des stratégies de réflexion, d'auto-évaluation et de partage des leçons apprises pour améliorer la pratique générale (Van der Wiele et Brown, 1999; Hockings et coll., 2006a, b). Cette approche est cohérente avec la construction d'une communauté de pratique où les participants cherchent à soutenir et apprendre les uns des autres par la co-création et l'échange de connaissances à travers des processus transparents de discussion qui impliquent divers systèmes de connaissances et révèlent de nouvelles perspectives (Sinclair et coll., 2008).

Dans cette perspective, notre équipe adopte un modèle d'apprentissage social caractérisé par " la collaboration, la confiance, l'exploration participative et partagée " (Glasser, 2007 : 51). De cette façon, les activités de recherche supportent et renforcent la capacité de tous les participants dans trois domaines clés : «l'évaluation critique des connaissances existantes et des problèmes, la production de connaissances [...] et l'application de ces nouvelles connaissances politiques et pratiques [...]» (Glasser, 2007 : 51). Le projet contribue à l'apprentissage social par le développement de compétences pratiques et de communication, et par l'examen des hypothèses sousjacentes à la prise d'action (Cranton, 2006 ; Taylor, 2007 ; Sipos et coll., 2008 ; Plummer, 2009). L'établissement de partenariat y est crucial, car il facilite l'implantation d'une communauté de pratique caractérisée par des participants qui désirent apprendre les uns des autres.

13 Les approches utilisées vont permettre la traduction des résultats des recherches en de nouvelles découvertes potentielles, la mise en réseau des chercheurs et des praticiens, l'émergence de stratégies d'évaluation appropriées et de mécanismes de mobilisation des connaissances pour les intervenants communautaires, universitaires et politiques à l'échelle nationale et internationale. Les étapes de démarche de recherche-action sont présentées à la figure 1 .

\section{Résultats préliminaires de la première année}

Au départ, l'idée d'un projet de création de réseau et de stratégies d'apprentissage sociale a suscité des réactions diverses et des inquiétudes chez les participants praticiens (coordonnateurs des réserves de biosphère): " [...] rien de concret ni véritablement bénéfique ne sortira de cet exercice académique ", mais somme toute, majoritairement positives, «[...] partager nos idées et installer la communication; apprendre les uns des autres; faire partie d'un réseau, ne plus être isolé; créer et 
partager une vision, un plan d'action, des objectifs communs; sentiment d'appartenance ».

Sur le plan pratique, les enjeux méthodologiques étaient et sont encore nombreux : comment intéresser et réunir les 16 réserves de biosphère, comment amorcer un dialogue de savoirs compte tenu de la l'immense étendue du pays, la diversité des paysages, des approches, des langages (pratique/théorique), des intérêts, des ressources financières réduites et des enjeux abordés localement. Grâce au financement, les participants se sont réunis et ont consacré une journée entière (jumelée à l'assemblée générale annuelle) à la mise en place de la démarche de recherche-action et à l'identification de thèmes de recherche. Le point de départ des discussions et des réflexions, proposé par les chercheurs, consistait en une question: quelles bonnes pratiques voulons-nous voir en place dans les trois prochaines années (réseautage, gouvernance, apprentissage) ? Au terme de la journée (idéation et ateliers de travail) les participants praticiens ont exprimé leur inconfort face à cet exercice et à la tangente qu'allait prendre la recherche-action. En réunion extraordinaire, le soir même autour d'un feu de camp et le lendemain midi, debout en cercle de discussion, au soleil sur la véranda, les praticiens ont exprimé librement leur vision aux chercheurs. La question de recherche proposée se transformait alors: qui sommes-nous individuellement et collectivement? Que faisons-nous? Quelles bonnes pratiques avons-nous? Ainsi, par le biais de cette appropriation du projet par les praticiens, naissait naturellement un agenda partagé dont le nouveau point de départ consistait à réaliser un inventaire de tous les projets des réserves de biosphère du Canada. À partir de cet inventaire (près de 430 projets), les praticiens ont commencé à découvrir leurs pairs et à identifier des similitudes (intérêts, projets). À l'aide d'un exercice de chevauchement des principaux champs d'intérêt (conservation, éducation développement et tourisme durable, aménagement du territoire et services écologiques, biodiversité monitoring et cartographie), les réserves de biosphère ont fait des alliances et formé des équipes de travail. Trois groupes ont été créés sur les thèmes suivants: tourisme durable, aménagement du territoire et services écologiques, et éducation. 


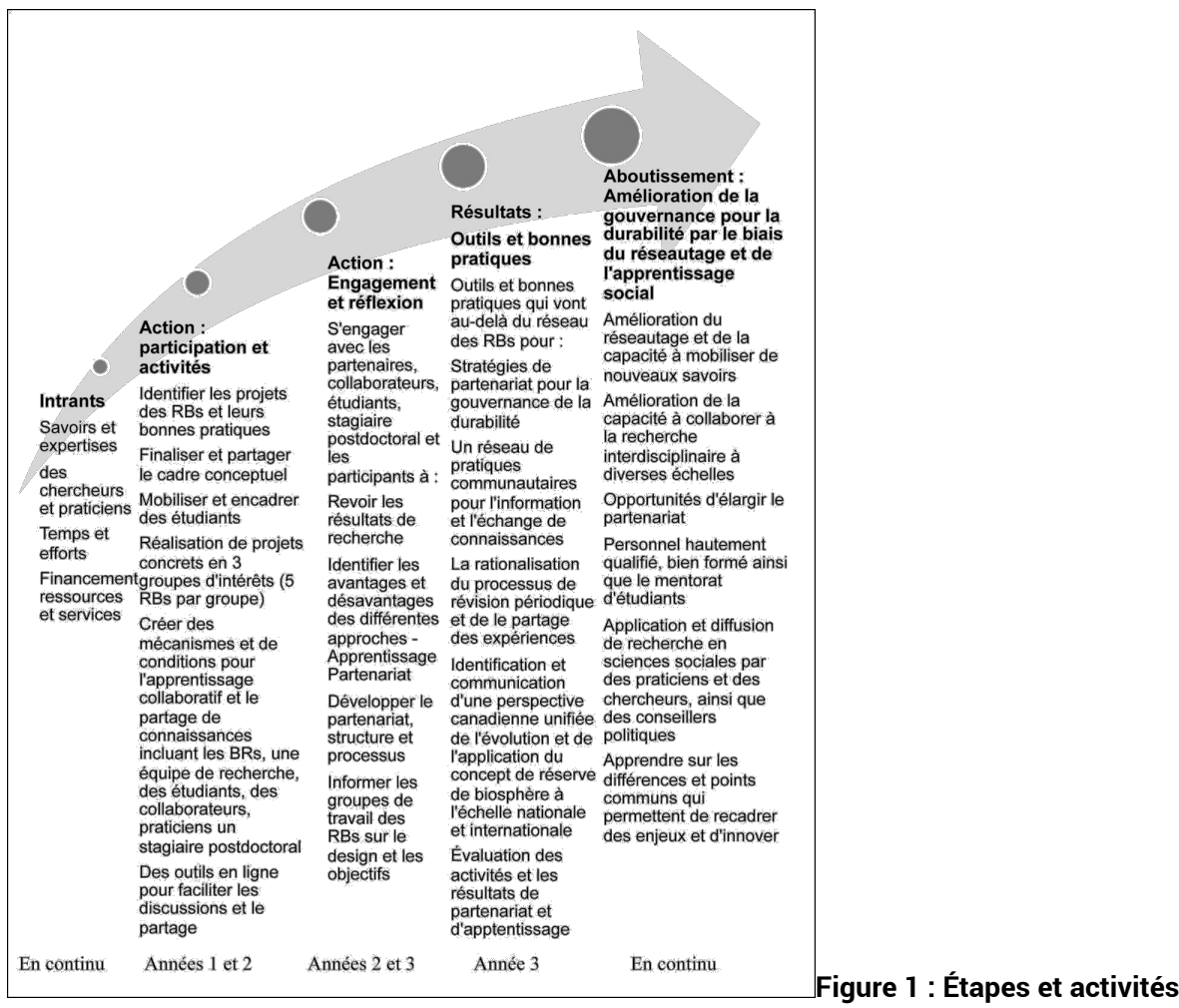

déployées dans le cadre de la recherche-action du projet de Création de réseaux et de stratégies d'apprentissage dans les réserves de la biosphère canadiennes

Ces groupes accompagnés des chercheurs ont eu la possibilité de se réunir physiquement une fois au cours de la première année. Lors de ces rencontres (incluant préparatif et suivi), un travail de partage d'expérience, d'introspection, d'analyse et d'évaluation des pratiques des réserves de biosphère a débuté. La contribution des chercheurs a pris la forme d'animation, d'accompagnement, d'observation et de formation sur mesure. Ces moments d'intense discussion et d'apprentissage partagé (parfois déroutants!) ont permis un véritable croisement des savoirs. Le travail de coformation et de co-construction des bonnes pratiques se poursuit encore aujourd'hui ; les échanges se font à l'aide de divers moyens de communication (conférence téléphonique, Skype, courriels, etc.).

17 Jusqu'à présent, les résultats sont encourageants. Les groupes ont réalisé ou sont en voie de terminer les réalisations et productions suivantes: 1) Groupe du Tourisme durable : 3 études des cas, un guide général, une boîte à outils, l'adhésion au Réseau international de tourisme durable, une participation au comité international MABUNESCO sur le même thème, un projet provincial financé (Ontario) stimulant le tourisme durable, 2) Groupe Aménagement du territoire et services écologiques : 5 études de cas, une analyse des éléments critiques (modèle), un état de l'usage des services écologiques dans les provinces, une boîte à outils sur les services écologiques et une offre de formation sur les techniques de facilitation de rencontres, 3) Éducation : un mini-guide d'éducation au développement durable, huit bonnes pratiques (cas), 12 exemples de projets, une boîte à outils (en ligne), une vidéo explicative du concept (en ligne) associée à un ensemble d'activités proposées pour découvrir les réserves de biosphère.

18 En même temps, le travail de recherche sur le processus et le développement de connaissances se poursuivent. Au centre de cette démarche se trouve le dénominateur 
commun que sont les bonnes pratiques. Autour de cet élément, les praticiens et les chercheurs s'approprient et déploient l'analyse critique et l'évaluation des rapports entre les savoirs scientifiques et les savoirs expérientiels. La démarche solidement appuyée sur les principes de l'apprentissage social, de la recherche participative, du réseautage et du partenariat assure un contexte stimulant qui évoluera idéalement vers une communauté de pratique.

\section{Conclusion}

Les valeurs promues par l'UNESCO, le Programme MAB et les missions des réserves de biosphère continuent d'être inspirantes. Cette étude permettra de mettre en évidence la richesse, la pertinence et le caractère innovateur des réalisations des réserves de biosphère depuis 40 ans, en dépit de leur relative précarité financière.

La volonté de comprendre et de poursuivre le développement de savoirs théoriques (pour l'humanité) d'une part, croisée aux besoins pratiques des réserves de biosphère de réaliser efficacement leur mission et les orientations de l'UNESCO, voire survivre, ouvre la voie à l'identification et la création de mécanismes de gouvernance et d'apprentissage intégrés.

Soulignons en terminant que les approches et pistes d'actions suggérées ci-dessus recoupent de façon évidente des éléments de pratiques de l'éducation relative à l'environnement et de l'éducation au développement durable (soit la recherche participative et la recherche-action). Il serait certainement enrichissant d'explorer plus en détail les éléments communs.

\section{BIBLIOGRAPHIE}

Abrams, P.E. (2000). Overcoming obstacles to implementing community-based collaborative governanceof natural resources : The case of the Clayoquot Sound Central Regional Board.

Unpublished Master Thesis. School of Resource and Environmental Management. Vancouver : Simon Fraser University.

Armitage, D., Marschke, M. et Plummer, R. (2008). Adaptive co-management and the paradox of learning. Global Environmental Change, 18(1), 86-98.

Bellamy, J.A., Walker, D.H., McDonald, G.T. et Syme, G.J. (2001). A systems approach to the evaluation of natural resource management initiatives. Journal of Environmental Management, $63,407-423$.

Berkes, F. (2010). Devolution of environment and resources governance : trends and future. Environmental Conservation, 37(3), 1-12.

Bevir, M. (2010). Democratic Governance. Princeton, NJ : Princeton University Press. Bouamrane, M. (dir.). (2007). Dialogue in biosphere reserves : references, practices and experiences. Biosphere reserves Technical Notes 2. Paris : UNESCO. 
Conley, A. et Moote, M.A. (2003). Evaluating collaborative natural resource management. Society and Natural Resources, 16, 371-386.

Cranton, P. (2006). Understanding and promoting transformative learning : A guide for educators of adults. San Francisco, CA : Jossey-Bass Publishers.

Dobell, R. (2002). Devolution and discretion : Building community-based resource management into contemporary governance. Dans Langford, J. et Edwards, M. (dir.). New players, partners, and boundaries : A public sector without borders? Canberra : National Institute.

Folke, C., Colding, J. et Berkes, F. (2003). Synthesis : building resilience and adaptive capacity in social-ecological systems. Dans Berkes, F., Colding, J. et Folke, C. (dir.). Navigating SocialEcological Systems : Building Resilience for Complexity and Change (p. 352-387). Cambridge, UK : Cambridge University Press.

Folke, C., Hahn, T., Olsson, P. et Norberg, J. (2005). Adaptive governance of social-ecological systems. Annual Review of Environment and Resources, 30, 441-473.

Francis, G. (2004). Biosphere reserves in Canada : Ideals and some experience. Environments, 32(3), 3-26.

Glasser, H. (2007). Minding the gap : The role of social learning in linking our stated desire for a more sustainable world to our everyday actions and policies. Dans Wals, A. (dir.). Social learning towards a sustainable world: Principles, perspectives, and praxis (p. 35-61). Wageningen, The Netherlands : Wageningen Academic Publishers.

Gunderson, L.H. et Holling, C.S. (2002). Panarchy : Understanding Transformations in Human and Natural Systems. Washington, DC : Island Press.

Hockings, M., Leverington, F. et James, R. (2006a). Evaluating management effectiveness. Dans Lockwood, M., Worboys, G.L. et Kothan, A. (dir.). Managing Protected Areas : A Global Guide. Camden, UK : Earthscan.

Hockings, M., Stolton, S., Leverington, F., Dudley, N. et Courrau, J. (2006b). Evaluating Effectiveness : A Framework for Assessing Management Effectiveness of Protected Areas, 2nd ed. Gland/Switzerland/Cambridge : IUCN/WCPA/WRI.

Jamieson, G., Francis, G., Whitelaw, G. et Ruttan, N. (2008). Canadian biosphere reserve approaches to the achievement of sustainable development. International Journal of Environment and Sustainable Development, 7(2), 132-144.

Keen, M., Brown, V.A. et Dyball, R. (dir.). (2005). Social learning in environmental management : Towards a sustainable future. London : Earthscan.

Löf, A. (2010). Exploring adaptability through learning layers and learning loops. Environmental Education Research, 16(5-6), 529-543.

Lotze-Campen, H., Reusswig, F. et Stoll-Kleeman, S. (2008). Socioecological monitoring of biodiversity change : building upon the world network of BRs. GAIA, 11(1), 107-115.

McCarthy, D., Whitelaw, G., Jongerden, P. et Craig, B. (2006). Sustainability, social learning, and the Long Point Biosphere Reserve. Environments 34(2), 1-15.

Mendis, S. (2004). Assessing community capacity for ecosystem management : Clayoquot Sound and Redberry Lake biosphere reserves. Unpublished Master Thesis. Saskatoon : University of Saskatchewan. 
Mendis-Millard, S. et Reed, M.G. (2007). Understanding community capacity using adaptive and reflexive research practices : Lessons from two Canadian biosphere reserves. Society and Natural Resources, 20(6), 543-559.

Pahl-Wostl, C. (2009). A conceptual framework for analysing adaptive capacity and multi-level learning processes in resource governance regimes. Global Environmental Change, 19, 354-365.

Plummer, R. et Armitage, D. (2010). Adaptive Capacity and Environmental Governance. Heidelberg : Springer Veriag.

Plummer, R. et Armitage, D. (2007). A resilience-based framework for evaluating adaptive comanagement : Linking ecology, economic and society in a complex world. Ecological Economics, 61, 62-74.

Plummer, R. (2009). The Adaptive Co-Management Process : an Initial Synthesis of Representative Models and Influential Variables. Ecology and Society, 14(2), art 24.

Pollock, R.M. (2004). Identifying principles for place-based governance in biosphere reserves. Environments, 32, 27-41.

Pollock, R.M (2009). The role of UNESCO biosphere reserves in governance for sustainability : Cases from Canada. Unpublished PhD Thesis. Peterborough, ON : Trent University.

Pollock, R.M., Reed, M.G. et Whitelaw, G.S. (2008). Steering governance through regime formation at the landscape scale : Evaluating experiences in Canadian biosphere reserves. Dans Hanna, K.S., Clark, D.A. et Slocombe, D.S. (dir.). Transforming parks and protected areas : Policy and governance in a changing world (p. 110-133). New York : Routledge.

Price, M.F., Park, J.J. et Bouamrane, M. (2010). Reporting progress on internationally designated sites : the periodic review of Biosphere Reserves. Environmental Science and Policy, 13, 549-557.

Reed, M.G. (2007). Uneven environmental management : A Canadian comparative political ecology, Environment and Planning A, 39, 320-338.

Réseau européen de la microfinance (REM). (2012). Qu'est-ce que les bonnes pratiques ? Récupéré le 12 octobre 2012 du site : www.european-microfinance.org/good-practice-definition.php

Reed, M.G. (2010). Creating networking and social learning strategies in Canadian biosphere reserves. Social Sciences and Humanities Research Council of Canada Partnership Development Grant.

Reed, M.G. et Egunyu, F. (2012). Management effectiveness in UNESCO Biosphere Reserves : Learning from Canadian periodic reviews. Environmental Science and Policy, 25, 107-117

Reed, M.G., Gibson, R. et Francis, G. (2008). Environmental governance for sustainability and resilience : Innovations in Canadian biosphere reserves and model forests. Funded Research Proposal to the Social Sciences and Humanities Research Council of Canada. Available from applicants, pp. 29

Roots, J.M. (1989). Biosphere reserves in Canada : Current status and future prospects. Unpublished.

Schliep, R. et Stoll-Kleemann, S. (2010). Assessing governance of biosphere reserves in Central Europe. Land Use Policy, 27, 917-927.

Schultz, L. et Lundholm, C. (2010). Learning for resilience ? Exploring learning opportunities in biosphere reserves. Environmental Education Research, 16(5), 645-663. 
Sian, S. (2000). The quest for functional sustainability in Canadian biosphere reserves : A process companion. Unpublished Master Thesis. Faculty of Environmental Design. Calgary, AB : University of Calgary.

Sipos, Y., Battisti, B. et Grimm, K. (2008). Achieving transformative sustainability learning : Engaging head, hands and heart. International Journal of Sustainability in Higher Education, 9(1), $68-86$.

Stoll-Kleemann, S. et Welp, M. (2008). Participatory and integrated management of biosphere reserves - Lessons from case studies and a global survey. GAIA - Ecological Perspectives for Science and Society, 17(S1), 161-168.

Stoll-Kleemann, S., de la Vega-Leinert, A.C. et Schultz, L. (2010). The role of community participation in the effectiveness of UNESCO Biosphere Reserve management : Evidence and reflections from two parallel global surveys. Environmental Conservation, 37(3), 227-238.

Taylor, E.W. (2007). An update of transformative learning theory : A critical review of the empirical research (1999-2005). International Journal of Lifelong Education, 26(2), 173-191.

UNESCO. (2013). Le Programme MAB : Le programme de l'UNESCO sur l'Homme et la biosphère. Récupéré le 12 octobre 2012 du site : www.unesco.org/new/fr/natural-sciences/environment/ ecological-sciences/man-and-biosphere-programme/

Whitelaw, G., Craig, B., Jamieson, G. et Hamel B. (2004). Research, monitoring and education : Assessing the "logistic function" of four Canadian biosphere reserves. Environments, 32(3), 61-78.

\section{NOTES}

1. Financé par le CRSNG - Programme de développement de partenariat.

2. Le concept de gouvernance fait référence ici aux arrangements formels et informels des prises de décisions qui impliquent des gouvernements et d'autres groupes sociaux (par exemple, le secteur privé, les communautés autochtones, les organisations de la société civile). Plus largement, la gouvernance inclut tous ceux qui contribuent à la prise de décision et qui sont en mesure de décider (Bevir, 2010).

\section{AUTEURS}

\section{HÉLÈNE GODMAIRE}

Hélène Godmaire est titulaire d'une maîtrise et d'un doctorat en biologie. Elle est également diplômée du programme court de 2e cycle en éducation relative à l'environnement de l'UQAM. Son intérêt et son implication dans des projets environnementaux scientifiques et communautaires l'amènent à travailler comme chercheure et consultante pour des universités, des ministères, des municipalités, des organismes environnementaux, des entreprises et des musées. Elle s'intéresse particulièrement à l'interdisciplinarité, la collaboration, la résilience et l'innovation. 


\section{MAUREEN REED}

Maureen Reed est professeure et directrice adjointe de l'École d'environnement et de développement durable à l'Université de la Saskatchewan. Son vaste programme de recherche porte sur la « la mise en œuvre de la durabilité » à travers trois organisations au Canada : les forêts communautaires, les forêts modèles et les réserves de la biosphère de l'UNESCO. Elle mène à la fois des analyses historiques et contemporaines afin de mieux comprendre comment le développement durable a été interprété et appliqué au fil du temps. Elle s'intéresse particulièrement à la façon dont les résidents ruraux sont touchés et peuvent contribuer aux décisions et aux politiques qui affectent leur environnement, les moyens de subsistance et la qualité de vie.

\section{MARC-ANDRÉ GUERTIN}

Marc-André Guertin a une formation en sciences de l'Université du Québec à Montréal (UQAM) et de l'Université McGill et une maîtrise en sciences de l'environnement de l'UQAM. De plus, il détient une formation de 2e cycle en éducation relative à l'environnement (UQAM) et s'intéresse à la formation des décideurs à l'environnement. Ces expériences de travail l'ont amené à participer à plusieurs études de cas pour faciliter la prise de décision en conservation et en aménagement du territoire. 